\title{
In search for a disease-modifying treatment in irritable bowel syndrome
}

\author{
Hans Törnblom, Magnus Simrén
}

IBS continues to evade the efforts of medical science to translate knowledge of putative pathogenetic or pathophysiological mechanisms into successful pharmacological treatments. To some extent, laxatives and antidiarrhoeal medications are able to improve the erratic bowel habits associated with IBS, and recently linaclotides have been shown to relieve abdominal pain and constipation in IBS-C. ${ }^{1}$ Even so, in those experiencing treatment success this cannot be linked to a disease-modifying effect, since stopping the treatment leads to recurrence of symptoms.

From this point of view, a hot topic during the past decades has been the many reports of low-grade immune activation in subgroups of patients with IBS. ${ }^{2}$

Department of Internal Medicine \& Clinical Nutrition, Institute of Medicine, Sahlgrenska Academy, University of Gothenburg, Gothenburg, Sweden

Correspondence to Professor Magnus Simrén, Department of Internal Medicine \& Clinical Nutrition, Institute of Medicine, Sahlgrenska Academy, University of Gothenburg, Gothenburg SE-41345, Sweden; magnus.simren@medicine.gu.se
The early observations of GI infections as a risk factor for developing IBS, ${ }^{3}$ but also other functional GI disorders such as functional dyspepsia, ${ }^{4}$ has sent signals of hope for anti-inflammatory treatment as a promising approach for a subset of patients. Epidemiological observations of a reduced risk for IBS in users of oral steroids pointed in the same direction. ${ }^{5}$ The lack of a positive effect of prednisolone treatment in postinfectious IBS, except for reducing the number of lamina propria lymphocytes, ${ }^{6}$ was disappointing but has not hampered the conceptual interest. Instead, it has rather focused efforts into medications with a better possibility for long-term treatment such as the mast cell stabiliser ketotifen ${ }^{7}$ and even more interesting, 5-aminosalicylic acid in the form of mesalazine ${ }^{8}$ that might affect both mast cells and lymphocytes.

In this issue of Gut, two multicentre, double blind, randomised and placebocontrolled trials exploring the effects of 12 weeks' treatment with mesalazine in IBS show negative outcomes. As disappointingly as this might seem, both studies taken together show the many inherent problems in evaluating IBS therapy. First, the definition and subgrouping of IBS per se are problematic, and the inconsistent association with underlying pathogenetic and pathophysiological abnormalities. There is general agreement about the use of the Rome criteria for inclusion of patients into clinical trials, and subtyping based on the predominant bowel habit is suggested. The study coordinated in Bologna by Barbara et $a l^{9}$ included patients with IBS of all subtypes, while the Nottingham study coordinated by Spiller and colleagues ${ }^{10}$ included only patients with IBS with diarrhoea. Both have made relevant assumptions to do so when planning the study design, but as is often the case, assumptions based on GI symptoms to include a patient group with a relevant underlying pathophysiological abnormality, notoriously define nonhomogeneous groups of patients. In the end, neither the Bologna study that included all IBS subtypes nor the Nottingham study that included IBS with diarrhoea could demonstrate an effect of mesalazine on the primary endpoints. This may not be a big surprise to some, since examples of symptoms for being bad predictors of underlying pathophysiology are many. Visceral hypersensitivity, ${ }^{11}$ gut motor abnormalities ${ }^{12}$ and signs of immune activation ${ }^{2}$ considered to be important factors in IBS can only be detected in a subset of patients with IBS, and mostly with modest associations with the symptoms reported by patients. 
Therefore, it may be better in future studies to base inclusion of patients in clinical trials in IBS on fulfilment of diagnostic criteria for IBS combined with detectable abnormalities of relevance for the pharmacological agent that is evaluated. However, the lack of effect of mesalazine on immune parameters in the Nottingham trial does not support that inclusion of patients with IBS with low-grade inflammation/immune activation in the GI tract would have altered the outcome substantially, even though other studies have found an effect of mesalazine on immune parameters in the GI tract in patients with IBS. ${ }^{8} 13$ From a theoretical point of view, it seems logical to only include patients who have the abnormality you want to treat with your drug.

The second inherent problem that might seem difficult to understand is that patients to include in clinical studies are difficult to find even though IBSs affect about $11 \%$ of the adult population across the globe. ${ }^{14}$ The studies in the current issue of Gut are examples of this, since slow recruitment necessitated changed study set-up in one and multiple study centres in the other. The difficulties in finding suitable patients for studies in a very common condition, to some extent, question if study results are applicable to the patients with IBS who visit gastroenterology and primary care outpatient clinics so frequently? This difficulty is by no means unique to these studies, but reported by many researchers. Study endpoints are another challenge in IBS, where there unfortunately is little consensus and recommendations change over time. This is exemplified by the two studies in this issue of Gut, which use different endpoints, making them difficult to compare with each other and also with studies assessing other agents. A big problem in IBS research is the lack of a valid objective measure as primary endpoint. The attempt to include a marker of immune activity such as mast cell or lymphocyte count in gut mucosa is probably important and relevant for this type of studies, but needs to be done consistently, and in larger sets of patients.

The disappointing results in these two much-awaited studies of course dampens the enthusiasm for mesalazine treatment in IBS. However, some clues how to move forward in order to potentially find a subgroup of patients with IBS who will respond to mesalazine or other antiinflammatory drugs are highlighted in the studies by Barbara et $a l^{9}$ and Lam et $a l,{ }^{10}$ which warrant further investigations. A fraction of patients do seem to respond in a meaningful and different way compared with placebo demonstrated by the explorative variation in responder definition made by Barbara et al, where it seems that a small subset of patients seem to respond if you use more strict endpoints. Defining a biomarker or patient characteristics that predict a greater chance for treatment response is an important task for future studies. Lam et al suggest that studying postinfectious IBS may be the way to go based on a trend towards a better response to mesalazine in that subgroup. Therefore, we believe that the current studies, despite being largely negative, provide ideas how to move forward in the area of anti-inflammatory treatments in IBS. To provide clinically meaningful results, inclusion probably still needs to be based on current diagnostic criteria for IBS, ideally combined with other features such as mode of onset of IBS or presence of immune activation/low-grade inflammation, but most importantly with collection of biological samples for analysis of immune activity, genetic polymorphisms associated with immune function and microbiota composition, combined with careful clinical phenotyping of patients in order to define the subset of patients that may respond to these drugs. As has been said after other negative clinical trials within GI, our interpretation of these two negative trials is that it is important not to throw out the baby with the bath water, ${ }^{15}$ but try to find the subset of patients with IBS that may respond favourably to mesalazine and other anti-inflammatories.

\section{Competing interests None declared.}

Provenance and peer review Commissioned; internally peer reviewed.

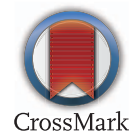

To cite Törnblom H, Simrén M. Gut 2016;65:2-3.

Received 22 May 2015

Accepted 25 May 2015

Published Online First 25 June 2015

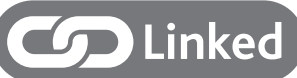

- http://dx.doi.org/10.1136/gutjnl-2014-308188

http://dx.doi.org/10.1136/gutjnl-2015-309122

Gut 2016;65:2-3. doi:10.1136/gutjnl-2015-310024

\section{REFERENCES}

1 Chey WD, Kurlander J, Eswaran S. Irritable bowel syndrome: a clinical review. JAMA 2015;313: 949-58.

2 Ohman L, Simren M. Pathogenesis of IBS: role of inflammation, immunity and neuroimmune interactions. Nat Rev Gastroenterol Hepatol 2010;7: 163-73.

3 Chaudhary NA, Truelove SC. The irritable colon syndrome. A study of the clinical features, predisposing causes, and prognosis in 130 cases. Q J Med 1962;31:307-22.

4 Mearin F, Perez-Oliveras M, Perello A, et al. Dyspepsia and irritable bowel syndrome after a Salmonella gastroenteritis outbreak: one-year follow-up cohort study. Gastroenterology 2005; 129:98-104.

5 Huerta C, Garcia Rodriguez LA, Wallander MA, et al. Users of oral steroids are at a reduced risk of developing irritable bowel syndrome. Pharmacoepidemiol Drug Saf 2003;12:583-8.

6 Dunlop SP, Jenkins D, Neal KR, et al. Randomized, double-blind, placebo-controlled trial of prednisolone in post-infectious irritable bowel syndrome. Aliment Pharmacol Ther 2003;18:77-84.

7 Klooker TK, Braak B, Koopman KE, et al. The mast cell stabiliser ketotifen decreases visceral hypersensitivity and improves intestinal symptoms in patients with irritable bowel syndrome. Gut 2010; 59:1213-21.

8 Corinaldesi R, Stanghellini V, Cremon C, et al. Effect of mesalazine on mucosal immune biomarkers in irritable bowel syndrome: a randomized controlled proof-of-concept study. Aliment Pharmacol Ther 2009;30:245-52.

9 Barbara G, Cremon C, Annese V, et al. Randomized controlled trial of mesalazine in irritable bowel syndrome. Gut 2016;65:82-90.

10 Lam C, Tan W, Leighton M, et al. A mechanistic multi-centre, parallel group, randomised placebo controlled trial of mesalazine for treatment of irritable bowel syndrome with diarrhoea (IBS-D). Gut 2016:65:91-9.

11 Tornblom H, Van Oudenhove L, Tack J, et al. Interaction between preprandial and postprandial rectal sensory and motor abnormalities in IBS. Gut 2014;63:1441-9.

12 Tornblom H, Van Oudenhove L, Sadik R, et al. Colonic transit time and IBS symptoms: what's the link? Am J Gastroenterol 2012;107: 754-60.

13 Castro Tejera V, Jerlstad $\mathrm{P}$, Venge $\mathrm{P}$, et al. A pilot study of mesalazine treatment in IBS, evaluating the tolerance and usefulness of the mucosal patch technique for measurement of recta inflammatory markers. Gastroenterology 2014; 146:S-542.

14 Lovell RM, Ford AC. Global prevalence of and risk factors for irritable bowel syndrome: a meta-analysis. Clin Gastroenterol Hepatol 2012; 10:712-21.e4.

15 Camilleri M, Acosta A. A ghrelin agonist fails to show benefit in patients with diabetic gastroparesis: let's not throw the baby out with the bath water. Neurogastroenterol Motil 2013; 25:859-63. 